\title{
Extension of structure groups of principal bundles in positive characteristic
}

\author{
Fabrizio Coiai and Yogish I. Holla
}

\begin{abstract}
In this article we study the behaviour of semistable principal $G$-bundles over a smooth projective variety $X$ under the extension of structure groups in positive characteristic. We extend some results of Ramanan-Ramanathan [20] on rationality of instability flags and show that the associated vector bundles via representations of $G$ are not too unstable and the instability can be bounded by a constant independent of semistable bundles. As a consequence of this the boundedness of the set of isomorphism classes of semistable $G$ bundles with fixed degree and Chern classes is proven.
\end{abstract}

Mathematics subject classification 14J60, 14L24

\section{Introduction}

Let $G$ be a connected reductive algebraic group over an algebraically closed field $k$ of arbitrary characteristic. Let $X$ be a smooth projective variety over $k$ with a fixed polarization $H$. In this paper we address the question of what happens to semistability of principal $G$-bundles under the extension of structure groups.

Recall the definition of a rational $G$-bundle $E$ as a principal $G$-bundle over a big open subscheme (whose complement is of codimension at least 2). A rational $G$-bundle $E$ over $X$ is semistable with respect to the polarization $H$ if for any reduction to a parabolic subgroup $P$ of $G$ over any big open subscheme, the line bundle associated to any dominant character on $P$ has degree $\leq 0$.

One notes that restrictions of torsion free sheaves to suitable open sets define rational GL( $V)$-bundles and in this case the above definition of semistability coincides with usual $\mu$-semistability.

Let $\rho: G \longrightarrow \mathrm{GL}(V)$ be a representation of $G$ on a vector space $V$ which sends the connected component of the center of $G$ to that of $\operatorname{GL}(V)$. For any rational $G$-bundle $E$ we denote by $E(V)$ the associated rational vector bundle.

When the characteristic of the field is zero, it is proved in [20] that the bundle $E(V)$ is semistable. If the characteristic of the field is a prime $p$ which is sufficiently large (quantified by the height of the representation) then the semistability of $E(V)$ is proved in [15]. 
In the case of arbitrary characteristic it is known that the bundle $E(V)$ need not be semistable. Let $\mu_{\max }(E(V))$ (and $\mu_{\min }(E(V))$ ) be the slopes of the first (and the last) term in the Harder-Narasimhan filtration of $E(V)$. We prove the following theorem.

Theorem 1.1 Let $\rho: G \rightarrow \mathrm{GL}(V)$ be a representation which sends the connected component of the center of $G$ to that of $\mathrm{GL}(V)$. Then there exists a constant $C(X, \rho)$ (depending only on $X$ and $\rho$ ) such that for each rational semistable $G$-bundle $E$ over $X$ we have

$$
\mu_{\max }(E(V))-\mu_{\min }(E(V)) \leq C(X, \rho)
$$

We briefly describe the proof. Let $E(G)$ be the group scheme associated to $E$ and $E(G)_{0}$ be the group scheme at the generic point $\operatorname{Spec}(k(X)) \rightarrow X$. Let $P$ be a maximal parabolic subgroup of $\mathrm{GL}(V)$ and let $\sigma$ be a rational reduction of structure group of $E(\mathrm{GL}(V))$. There is an action of $E(G)_{0}$ on the smooth projective variety $E(\mathrm{GL}(V) / P)_{0}$ over $k(X)$ which is linearized by a suitable line bundle. The section $\sigma$ gives a $k(X)$-valued point $\sigma_{0}$ of $E(\mathrm{GL}(V) / P)_{0}$. It is known that if $\sigma_{0}$ is a semistable point for the above action then the reduction $\sigma$ does not violate the semistability of $E(\mathrm{GL}(V))$ (see Proposition (3.8)). Also, if $\sigma_{0}$ is not semistable and its instability parabolic $P\left(\sigma_{0}\right)$ (see Section 3 for the definition) is defined over the function field $k(X)$ of $X$ then again $\sigma$ does not violate the semistability of $E(\mathrm{GL}(V)$ ) (see Proposition (3.9)). This argument in characteristic zero proves that $E(V)$ is semistable because $P\left(\sigma_{0}\right)$ is defined over $\overline{k(X)}$ and by its uniqueness it is invariant by the Galois group hence it is defined over $k(X)$.

In the case of characteristic $p$ one of the important points in our proof is to show that there is an integer $N$ (independent of the $G$-bundle $E$ ) such that if $\sigma_{0}$ is not semistable then its instability parabolic is defined over the field $k(X)^{p^{-N}}$ (see Proposition 4.5). This part is achieved by repeated use of an algebraic result which enables us to get uniform bounds for non-reducedness of fibers of morphisms of algebraic varieties (see Proposition 4.2).

Once the instability parabolic is defined over $K^{p^{-N}}(X)$, this parabolic gives rise to a reduction of structure group of the Frobenius pull-back $\left(F^{N}\right)^{*} E$. Using this reduction and some geometric invariant theory arguments, we reduce the problem to proving the following Theorem which bounds instability of Frobenius pull-backs.

Theorem 1.2 There exists a constant $C(X, G)$ and a constant $N(G)$ such that for any rational $G$-bundle $E$ we have

$$
\operatorname{Ideg}\left(F^{*} E\right) \geq p N(G) \operatorname{Ideg}(E)+C(X, G)
$$

(The instability degree Ideg is defined by equation (11) (in Section 2).

In the case of vector bundles, the above result was proved by X. Sun (see [23]) and Shephard-Barron (see [22]).

We use the Theorem (1.1) for groups of lower semisimple rank to prove the Theorem (1.2). In fact we prove a generalization of Theorem (1.1) where we replace GL $(V)$ 
by an arbitrary reductive group and (1.2) will then be a special case of this result (see Remark (5.5)).

Let $\widetilde{c}_{i} \in A^{*}(X)$ for $i=1 \ldots n$ be elements with $n=\operatorname{dim}(X)$. Let $S_{b}\left(r ; \widetilde{c}_{1} \ldots, \widetilde{c}_{n}\right)$ be the set of isomorphism classes of torsion free sheaves $V$ of rank $r$ and $c_{i}(V)=\widetilde{c}_{i}$ satisfying $\mu_{\max }(V)-\mu_{\min }(V) \leq b$.

Let $c_{i} \in A^{i}(X)$ for $2 \leq i \leq n$ be fixed. We also fix a homomorphism $d \in$ $\operatorname{Hom}\left(\mathcal{X}(G), A^{1}(X)\right)$. Here $A^{k}(X)$ 's are the Chow groups and $\mathcal{X}(G)$ is the group of characters.

In the last section, we use the above results to show the following result on boundedness of semistable $G$-bundles (which are defined on all of $X$ ).

Theorem 1.3 Assume that the set $S_{b}\left(r ; \widetilde{c}_{1}, \ldots, \widetilde{c}_{n}\right)$ is bounded for all choices of $\widetilde{c}_{i}, b$ and $r$. Then the set $\mathcal{S}_{G}\left(d ; c_{2}, \ldots, c_{n}\right)$ of isomorphism classes of semistable $G$-bundles $\{E\}$ with degree $d_{E}=d$ and $c_{i}(\operatorname{ad}(E))=c_{i}$ is bounded.

Here the degree of a principal bundle $E$ is an element $d_{E} \in \operatorname{Hom}\left(\mathcal{X}(G), A^{1}(X)\right)$ defined by $d_{E}(\chi)=c_{1}\left(\chi_{*}(E)\right)$ for any character $\chi$ of the group $G$. Here $\chi_{*}(E)$ is the line bundle associated to $E$ via $\chi$.

In characteristic 0 , the boundedness of the set $S_{b}\left(r ; \widetilde{c}_{1}, \ldots, \widetilde{c}_{n}\right)$ is well known (see [11 for example). In the case of positive characteristic, for surfaces, this is due to Gieseker [7 and Maruyama [16. For higher dimensional varieties this is recently claimed by Langer [14]. This along with Theorem (1.3) would then prove boundedness of semistable $G$-bundles over $X$ with fixed Chern classes.

When $X$ is a smooth projective curve in characteristic 0 , the boundedness of the semistable $G$-bundles with fixed degree is due to Ramanathan [19; in the case of positive characteristic, it is proved in [10].

Acknowledgments The authors wish to thank M.S. Narasimhan who initiated them into this problem, and also for sharing his ideas. The first named author would like to thank the Tata Institute of Fundamental Research (Mumbai and Bangalore centers) for hospitality.

\section{Basic definitions and notations}

\section{Semistable $G$-bundles}

In this section we recall and prove some basic facts about principal $G$-bundles over varieties. Let $k$ be an algebraically closed field. Let $G$ be a connected reductive algebraic group over $k$. Let $T$ be a maximal torus and $B$ a Borel subgroup containing $T$. Let $R_{u}(B)$ be the unipotent radical of $B$. Then $B$ is a semi-direct product $R_{u}(B) \cdot T$. We denote by $\mathcal{X}_{*}(T)$, the group of 1-parameter subgroups of $T$ (denote by 1 -PS $). \mathcal{X}^{*}(T)$ denotes the group of characters of $T$. We have a perfect pairing $\mathcal{X}_{*}(T) \otimes \mathcal{X}^{*}(T) \longrightarrow \mathbb{Z}$ which will be denoted by $(\cdot, \cdot)$. Let $\Phi \subset \mathcal{X}^{*}(T)$ be the set of roots of $G, \Phi^{+}$be the set of positive roots and $\Delta$ be the set of simple roots 
corresponding to $B$. For any $\alpha \in \Phi$, let $T_{\alpha}$ be the connected component of $\operatorname{ker}(\alpha)$ and $Z_{\alpha}$ the centralizer of $T_{\alpha}$ in $G$. Then the derived group $\left[Z_{\alpha}, Z_{\alpha}\right]$ is of rank one and there is a unique 1-PS $\hat{\alpha}: \mathbb{G}_{m} \longrightarrow T \cap\left[Z_{\alpha}, Z_{\alpha}\right]$ such that $T=(\operatorname{im} \hat{\alpha}) \cdot T_{\alpha}$ and $(\hat{\alpha}, \alpha)=2$. This $\hat{\alpha}$ is the coroot corresponding to $\alpha$. We denote by $\Phi$ the set of coroots. The quadruple $\left\{\mathcal{X}^{*}(T), \Phi, \mathcal{X}_{*}(T), \hat{\Phi}\right\}$ defines a root system. For each $\alpha \in \Delta$ we have the fundamental dominant weight $w_{\alpha} \in \mathcal{X}^{*}(T) \otimes \mathbb{Q}$ defined by $\left(\hat{\beta}, w_{\alpha}\right)=\delta_{\alpha, \beta}$ for $\beta \in \Delta$ and $\left(\gamma, w_{\alpha}\right)=0$ for any 1-parameter group in the connected component of the center of $G$. Let $W=N(T) / T$ be the Weyl group. We fix a $W$-invariant inner product on $\mathcal{X}_{*}(T) \otimes \mathbb{Q}$ (hence on $\mathcal{X}^{*}(T) \otimes \mathbb{Q}$ ).

Let $P$ be a parabolic subgroup of $G$ containing $B$. Let $U$ be its unipotent radical.

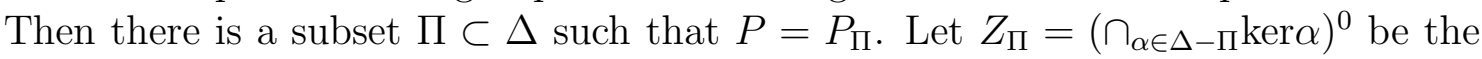
connected component of the intersection of kernels of roots in $\Delta-\Pi$. By taking the centralizer of $Z_{\Pi}$ one obtains a splitting $P \rightarrow P / U=L$ with $Z_{\Pi}$ being the connected component of the center of $L$.

Let $X$ be a smooth projective variety over $k$ of dimension $n$ and let $k(X)=K$ be the function field of $X$. Let $H$ be a fixed polarization on $X$. Since any line bundle $\mathcal{L}$ over a big open subscheme $U$ (whose complement is of codimension at least 2) admits a unique extension to all of $X$, its first Chern class makes sense. Recall the definition of the degree of the line bundle to be $\operatorname{deg}(L)=c_{1}(L) \cdot H^{n-1}$. Hence for any torsion free sheaf its first Chern class and the degree with respect to $H$ makes sense.

We recall from [20] that a rational $G$-bundle $E$ is a principal $G$-bundle over a big open subscheme of $X$. For $G=\mathrm{GL}(V)$ this defines a vector bundle over a big open subscheme and we call it rational vector bundle.

Let $E \longrightarrow U \subset X$ be a rational $G$-bundle with $U$ a big open subscheme. By rational reduction of structure group $\sigma$ of $E$ to $P$ we mean a reduction of structure group over a big open subscheme $U^{\prime} \subset U$. More precisely it is a pair $\left(E_{\sigma}, \phi\right)$ with $E_{\sigma}$ a $P$-bundle over $U^{\prime}$ and an isomorphism $\phi:\left.E_{\sigma}(G) \longrightarrow E\right|_{U^{\prime}}$. This is equivalent to giving a section $\sigma$ of the fiber bundle $\pi: E / P \longrightarrow U$ over $U^{\prime}$. Here $E / P$ denotes the extended rational fiber bundle $E(G / P)$ over $X$.

Let $T_{\pi}$ be the tangent bundle along the fibers of the map $\pi$. Then $T_{\pi}$ is a rational vector bundle. For a reduction of structure group $\sigma$ we will denote by $T_{\sigma}$ the rational vector bundle defined by the pull-back of $T_{\pi}$ under $\sigma$. We will also fix notations for the Lie algebras by putting $\mathfrak{g}, \mathfrak{p}$ and $\mathfrak{l}$ for the Lie algebras of $G, P$ and $L$ respectively. Then it can be verified that $T_{\sigma}$ is the rational vector bundle on $X$ associated to $E_{\sigma}$ for the natural representation of $P$ on $\mathfrak{g} / \mathfrak{p}$.

Recall the following definition of semistability from Ramanan-Ramanathan [20].

Definition 2.1 A rational G-bundle $E \longrightarrow U \subset X$, with $U$ a big open set is semistable with respect to polarization $H$ if for any reduction of $E$ to any parabolic subgroup $P$ of $G$ over any big open set $U^{\prime} \subset U$, the line bundle associated to any dominant character on $P$ has degree $\leq 0$.

This definition is equivalent to the fact that $\operatorname{deg}\left(T_{\sigma}\right)>0$ for each rational parabolic reduction. 
If $V$ is a torsion free sheaf then over a big open set $U$ the restriction $\left.V\right|_{U}$ is a vector bundle. The above definition of semistability is equivalent to the $\mu$-semistability of $V$.

\section{Instability degree and Harder-Narasimhan reduction}

If the rational $G$-bundle is not semistable then there is a notion of Harder-Narasimhan reduction which we recall here. For a rational $G$-bundle $E$ which is not semistable we define the instability degree to be:

$$
\operatorname{Ideg}(E)=\operatorname{Min}_{\{P, \sigma\}} \operatorname{deg}\left(T_{\sigma}\right)
$$

where the minimum is taken over all parabolic subgroups $P$ and rational reductions $\sigma$. If the rational $G$-bundle is semistable then we say its instability degree is 0 . The following lemma shows that the instability degree makes sense, and is an analogue of Lemma 2.1 of [10] for the higher dimensional varieties.

Lemma 2.2 There exists a constant $A_{E}$ such that for any rational reduction $\sigma$ of $E$ to any parabolic $P$ we have $\operatorname{deg}\left(T_{\sigma}\right)>A_{E}$

Proof It is enough to show that the degree of the rational vector subbundle $\operatorname{ad}\left(E_{\sigma}\right) \subset \operatorname{ad}(E)$ is bounded above.

We can first extend the bundle $\operatorname{ad}(E)$ to get a torsion free sheaf $\mathcal{E}$ on $X$. Then we can extend $\operatorname{ad}\left(E_{\sigma}\right)$ inside $\mathcal{E}$ to obtain a torsion free subsheaf. There exists a constant $A_{E}^{\prime}$ such that for any curve $C$ in the class $\left|H^{n-1}\right|$, we have a bound $h^{0}\left(C,\left.\mathcal{E}\right|_{C}\right) \leq A_{E}^{\prime}$. Let $g$ be the maximum of the genus of smooth curves in $\left|H^{n-1}\right|$. Now if $C$ is a smooth projective curve which sits in the domain of definition of $\operatorname{ad}\left(E_{\sigma}\right)$ and $\operatorname{ad}(E)$ then we get $\operatorname{deg}\left(E_{\sigma}\right) \leq A_{E}^{\prime}+(g-1) \operatorname{rank}(\operatorname{ad}(E))$. This proves the lemma.

Definition 2.3 A rational reduction of structure group $\sigma$ of $E$ to a parabolic $P$ is said to be a Harder-Narasimhan reduction if $\operatorname{deg}\left(T_{\sigma}\right)=\operatorname{Ideg}(E)$ and $P$ is maximal among parabolic subgroups of $G$ containing $B$ for which the above equality holds.

The Harder-Narasimhan reductions as defined above satisfy the following properties stated in Ramanathan [21]. (see [4] for a proof).

1. If $L$ is the Levi quotient of $P$, then the principal $L$-bundle $E_{\sigma}(L)$ obtained by extending the structure group is semistable;

2. After fixing a Borel subgroup $B \subset P$ of $G$, for any nontrivial character $\chi$ of $P$ which is a non-negative linear combination of simple roots, the associated rational line bundle $\chi_{*}\left(\left(E_{\sigma}\right)\right)$ over $X$ is of positive degree. 
It is proved in Behrend [3] that over a smooth projective curve there is a unique reduction to a parabolic subgroup containing $B$ satisfying the above properties. In the case when $X$ is higher dimensional the uniqueness is known only when the characteristic of the field is 0 or it is a large prime $p$.

We will not have the occasion to use the uniqueness of the above reduction. We will only use its existence.

For the case $G=\mathrm{GL}(V)$ the above reduction defines the Harder-Narasimhan filtration and the uniqueness is then immediate. We have the following lemma which compares the instability degree with the $\mu_{\max }-\mu_{\min }$ of the rational vector bundle.

Lemma 2.4 Let $E$ be a rational principal $\mathrm{GL}(V)$ bundle of rank $r$ over $X$ which is not semistable (we will denote by $E(V)$, the associated vector bundle). Then we have the following.

$$
\mu_{\max }(E(V))-\mu_{\min }(E(V)) \leq-\frac{2}{r^{2}} \operatorname{Ideg}(E)
$$

Proof For the proof one first notices that if $F \subset E(V)$ is a rational subbundle of rank $r_{1}$ and $F_{1}$ is the quotient, then it defines a rational reduction of structure group $\sigma$ of $E$ to a maximal parabolic $P_{1}$ of $\mathrm{GL}(V)$. One further has an isomorphism of rational bundles $T_{\sigma} \cong \mathcal{H o m}\left(F, F_{1}\right)$.

This implies that $\mu(F)-\mu\left(F_{1}\right)=-\mu\left(\mathcal{H o m}\left(F, F_{1}\right)\right) \leq-\operatorname{Ideg}(E) /\left(r_{1}\left(r-r_{1}\right)\right)$.

This inequality can also be written by eliminating $F_{1}$ or $F$, we get $\mu(F)-\mu(E(V)) \leq$ $-\operatorname{Ideg}(E) /\left(r r_{1}\right)$ and $\mu(E(V))-\mu\left(F_{1}\right) \leq-\operatorname{Ideg}(E) /\left(r\left(r-r_{1}\right)\right)$.

Now if we take $F$ to be the rational subbundle which is maximal destabilizing then we have $\mu_{\max }(E(V))=\mu(F)$. This implies that $\mu_{\max }(E(V))-\mu(E(V)) \leq$ $-\operatorname{Ideg}(E) / r^{2}$. Similarly one has $\mu(E(V))-\mu_{\min }(E(V)) \leq-\operatorname{Ideg}(E) / r^{2}$. Combining these we have the proof of the lemma.

\section{Frobenius morphism}

Let $k$ be an algebraically closed field of characteristic $p>0$. The $p^{m}$-th power map $\mathcal{O}_{X} \rightarrow \mathcal{O}_{X}$ given by $f \rightarrow f^{p^{m}}$ is a homomorphism and gives rise to a morphism $F_{X}^{m}: X \rightarrow X$ called the absolute Frobenius morphism. Using this morphism we can pull-back a $G$-bundle $E$ over $X$ to get a $G$ bundle $\left(F_{X}^{m}\right)^{*}(E)$ which we will denote by $\left(F^{m}\right)^{*} E$. One notes that the absolute Frobenius morphism is not a $k$-morphism. But since $k$ is a perfect field we can always twist by the Frobenius isomorphism of $k$ to ensure that the $G$-bundle $\left(F^{m}\right)^{*}(E)$ has a well defined $k$-structure.

We will use the following well known result about Frobenius morphisms.

Proposition 2.5 Let $E$ be a rational $G$-bundle over $X$. Then there exists a $p$ connection $\nabla$ on the $G$-bundle $F^{*}(E)$ which satisfies the following property: for any rational reduction of structure group $\sigma$ of $F^{*}(E)$ to a parabolic $P$ there is a vector bundle map (second fundamental form) $\nabla_{\sigma}: T_{X} \rightarrow T_{\sigma}$ (wherever $T_{\sigma}$ is defined) such that the following are equivalent. 
1. There exists a rational reduction $\sigma_{0}$ of $E$ to $P$ such that $F^{*}\left(\sigma_{0}\right)=\sigma$.

2. $\nabla_{\sigma}$ is zero.

Proof See for example, proof of Theorem (2.1) of [23].

\section{Geometric invariant theory and the method of Ramanan and Ramanathan}

In this section we will describe some basic facts about geometric invariant theory and briefly explain the results of Ramanan and Ramanathan [20].

\section{The instability parabolic}

Let $K$ be a field and let $\bar{K}$ be a fixed algebraic closure. Let $G$ be a connected reductive algebraic group over $K$. Let $V$ be a finite dimensional representation of $G$ we get an induced action of $G$ on the projective space $\mathbb{P}(V)$ of lines in $V$. For a point $v \in \mathbb{P}(V)$ we will denote, by abuse of notation, a representative in $V$ again by $v$.

Firstly we will describe the theory when $K=\bar{K}$ is algebraically closed and later extend the theory to non-algebraically closed fields.

Recall that a point $0 \neq v \in V$ is semistable for the $G$-action if the closure $\overline{G v}$ of the orbit of $v$ does not contain 0 . One knows that this definition is equivalent to existence of a $G$-invariant element $\phi \in S^{n}\left(V^{*}\right)$ for some $n>0$ such that $\phi(v) \neq 0$.

If $v \in V$ is not semistable then recall the following notions.

For a 1-PS $\lambda$ of $G$, consider the decomposition of $V=\bigoplus V_{i}$ with $V_{i}=\{v \in$ $\left.V \mid \lambda(t) v=t^{i} v\right\}$. For an $v \in V$ one defines the invariant

$$
m(v, \lambda)=\inf \left\{i \mid v \text { has a non-zero component in } V_{i}\right\}
$$

Using the $W$-invariant inner product on a fixed maximal torus $T$ one defines the slope $\nu(v, \lambda)=m(v, \lambda) /\|\lambda\|$ for all 1-PS in the maximal torus $T$. Since maximal tori are conjugates this definition can be extended to all 1-PS in $G$.

The following lemma will be used in the sequel.

Lemma 3.1 With the above notations there exists a constant $C$ (independent of $v \in V$ and $1-P S \lambda)$ such that $\nu(v, \lambda) \leq C$.

Proof See Proposition (2.17) p. 64 of [18] for a proof.

We define the instability 1-PS for a given $v \in V$ (which is not semistable) as one for which $\nu(v, \lambda)$ attains its maximum among all 1-PS of $G$ (see Theorem $(1.5$, a) of [20]). 
For a 1-PS $\lambda$, recall the definition of the parabolic $P(\lambda)$ whose valued points are

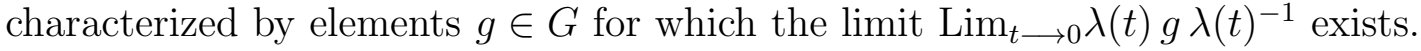

In the following Proposition we summarize the basic facts in geometric invariant theory.

Proposition 3.2 Let $v \in V$ be a non-zero element which is non-semistable.

1. There is a unique parabolic subgroup $P(v)$ with the property that for any instability 1-PS $\lambda$ for $v$ we have $P(v)=P(\lambda)$.

2. For any maximal torus $T \subset P(v)$ there is a unique 1-PS $\lambda_{T} \subset T$ such that it is an instability 1-PS for $v$.

Proof See Theorem (1.5, b and c) [20].

The above uniquely defined parabolic $P(v)$ will be called the instability parabolic for $v$. Here the uniqueness of $\lambda$ is as a subgroup of $T$ rather than a morphism $\mathbb{G}_{m} \rightarrow T$.

If $G$ acts on projective variety $M$ which is linearized by an ample line bundle $\mathcal{L}$ then by taking some power of $\mathcal{L}$ we get a representation $V$ of $G$ and a $G$-equivariant embedding $i: M \rightarrow \mathbb{P}(V)$ with $i^{*} \mathcal{O}(1)$ being some power of $\mathcal{L}$. In this setup we say a point $m \in M$ is semistable for $G$ action if the corresponding point in $V$ is semistable.

Let $v \neq 0$ be a non-semistable point. Let $P=P(v)$ be its instability parabolic and let $\lambda \subset T \subset P$ be a chosen tuple of instability 1-PS and a maximal torus $T$. Let $V=\bigoplus_{i} V_{i}$ be the decomposition of $V$ with respect to $\lambda$. Let $j=m(v, \lambda)$. Using this we have a decomposition $v=\sum_{i \geq 0} v_{i}$, where $v_{i} \in V_{i+j}$.

Here one notes that $V^{j}=\bigoplus_{i \geq j} V_{i}$ is preserved under the action of $P=P(v)$ and the unipotent radical $U \subset P$ pushes $V^{j}$ to $V^{j+1}$, thus giving an action of the Levi quotient $L=P / U$ on $V^{j} / V^{j+1}$.

The $W$-invariant inner product on a fixed maximal torus of $G$ naturally gives rise to a $W$-invariant inner product on $T \subset P(v)$. Let $l_{\lambda} \in \mathcal{X}^{*}(T) \otimes \mathbb{Q}$ be the dual of $\lambda$. Let $r_{1} \in \mathbb{Z}^{+}$such that $r_{1} l_{\lambda}$ defines a character of $T$. The restriction of this character to the connected component $Z^{0}(L)$ of the center of $L$, and taking a further multiple, extends to give a character of $L$. Hence given a $\lambda$ we get a character $\chi$ of $P(v)$ which is well defined up to a positive integral multiple.

In the following proposition we describe the basic result of Ramanan-Ramanathan [20] concerning the behaviour of $v_{0} \in V^{j} / V^{j+1}$ under the induced action of $L$ on $V^{j} / V^{j+1}$.

Proposition 3.3 Assume that the group $Z^{0}(G)$ acts trivially on $V$. Then there exists a positive integer $r$ and dominant character $\chi$ of $P$ such that the point $v_{0} \in$ $\mathbb{P}\left(V^{j} / V^{j+1}\right)$ is semistable for the natural action of $L$ with respect to linearization given by $\mathcal{O}(r) \otimes \mathcal{O}_{\chi^{-1}}$, where $\mathcal{O}_{\chi^{-1}}$ is the trivial line bundle with $L$ acting on it by $\chi^{-1}$ 
The proof of the above result (as given in Proposition (1.12) in [20]) also gives a recipe to find the integer $r$ and the character $\chi$ and they are related by the following Lemma.

Lemma 3.4 There is a character $\chi^{\prime}$ of the maximal torus $T \subset P$ such that the following holds.

$$
\begin{aligned}
& \text { 1. }\left.\chi^{\prime}\right|_{Z^{0}(L)}=\left.\chi\right|_{Z^{0}(L)} \\
& \text { 2. } \chi^{\prime}=r \nu(v, \lambda)\|\lambda\| l_{\lambda} \text {, where } l_{\lambda} \text { is the dual of } \lambda .
\end{aligned}
$$

The above Lemma will be used in our proof of the main Theorem.

\section{The Rationality of the instability parabolic}

We will now assume that the ground field $K$ is not algebraically closed. Let $G$ be a connected reductive group over $K$ which acts on a projective $K$-variety $M$, linearized by an ample line bundle $\mathcal{L}$, thus giving a $G$-equivariant embedding $i: M \rightarrow \mathbb{P}(V)$ as before.

We will call a $K$-valued point $v \in V$ semistable if it is so after a base change to algebraic closure. In this way we will avoid the confusion of which field the semistability definition is used.

Let $m$ be a $K$-rational point of $M$ which is not semistable. Let $P(m)$ be its instability parabolic defined over $\bar{K}$.

Remark 3.5 Note that if $P(m)$ is defined over $K_{s}$ then it is already defined over $K$. This is because of the uniqueness of $P(m)$ (see Proposition $3.2(1)$ ) and the Galois descent argument. Also note that if $P(m)$ is defined over $K$ then it contains a maximal torus over $K$ which splits over $K_{s}$. Then the instability 1-PS of $m$ which is contained in the maximal torus over $K_{s}$, by uniqueness (Proposition $3.2(2)$ ) is Galois invariant and hence it is defined over $K$.

Let $O(m)$ be the (reduced) orbit of $G$ at $m$. Since $m$ is defined over $K$ the orbit $O(m)$ is also defined over $K$.

We briefly recall the construction of a scheme $M(P)_{x_{m}}$ which will be used later in an important way.

We can find a $g \in G$ such that $g P(m) g^{-1}=P$ is defined over $K_{s}$ (as the variety of all parabolics conjugate to $P(m)$ is defined over $K_{s}$, being absolutely reduced, has a $K_{s}$-rational point (see [6])). If $x_{m}=g m$ then $P=P\left(x_{m}\right)$ is the instability parabolic of $x_{m}$.

Since $P$ is defined over $K_{s}$ and over this field $G$ splits, we have a maximal torus in $P$ which splits over $K_{s}$. Hence there is a instability 1-PS $\lambda$ of $x_{m}$ in this maximal torus over $K_{s}$.

The representation $V$ of $G$ decomposes as $V=\bigoplus_{i \in \mathbb{Z}} V_{i}$ for the action of $\lambda$, where $V_{i}=\left\{v \in V \mid \lambda(t) \cdot v=t^{i} v, t \in \mathbb{G}_{m}\right\}$. Let $j=m\left(x_{m}, \lambda\right)$ and $V^{j}=\bigoplus_{i \geq j} V_{i}$. 
Recall the definition of the $K_{s}$-scheme $M(P)_{x_{m}}$ as the scheme theoretic intersection of the $K_{s}$-subschemes $\mathbb{P}\left(V^{j}\right)$ and $O(m)$ of $\mathbb{P}(V)$.

The following two results summarizes the basic properties of the scheme $M(P)_{x_{m}}$.

Lemma 3.6 The $\bar{K}$-rational points of the $K_{s}$-subscheme $M(P)_{x_{m}}$ of the $K$-scheme $O(m)$ are precisely those points which have $P\left(x_{m}\right)$ as their instability parabolic. Moreover, when the $G$ action on $m$ is strongly separable then $M(P)_{x_{m}}$ is absolutely reduced.

Proof See Lemma (2.4) of [20].

Recall that the $G$-action at $m \in M(\bar{K})$ is said to be strongly separable if the isotropy subgroup scheme $G_{x}$ is reduced at every point $x \in M(\bar{K})$ which is in the closure of the orbit $O(m)$.

Lemma 3.7 Suppose that $y \in M(P)_{x_{m}} \subset O(m)$ is a $K_{s}$-rational point and that there is an $h \in G\left(K_{s}\right)$ such that $h$ maps to $y$ under the orbit map $G \rightarrow O(m)$. Then $P(m)$ is defined over $K$.

Proof By lemma (3.6), the point $y=h m$ has the property that $P(y)=P\left(x_{m}\right)$, hence $P(y)$ is defined over $K_{s}$. This implies that $P(m)=h P(y) h^{-1}$ is also defined over $K_{s}$, hence by Remark (3.5) we conclude the proof of the Lemma.

The above lemma has the consequence that if the action of $G$ is strongly separable at $m$ then the parabolic $P(m)$ is already defined over $K$ (also see Proposition 2.4, [20]).

\section{The argument of Ramanan and Ramanathan}

Let $E$ be a rational $G$-bundle over $X$. Let $\rho: G \longrightarrow G_{1}$ be a representation of $G$ which takes the connected component of the center of $G$ to the center of $G_{1}$. Let $P_{1}$ be a parabolic subgroup of $G_{1}$. We fix a representation $G_{1} \longrightarrow \mathrm{GL}\left(V_{P_{1}}\right)$ such that it defines an embedding of $G_{1} / P_{1} \subset \mathbb{P}\left(V_{P_{1}}\right)$ with the property that the character of $P_{1}$ on $V_{P_{1}}$ is a positive multiple $m_{P_{1}}$ of the character of $\chi_{P_{1}}$ associated to the restriction of the adjoint representation of $P_{1}$ on the vector space $\mathfrak{g}_{1} / \mathfrak{p}_{1}$.

The line bundle $\mathcal{O}(1)$ on the projective variety $\mathbb{P}(V)$ gives rise to a line bundle $\mathcal{L}$ over $G_{1} / P_{1}$.

For the rational $G$-bundle $E$ over $X$ we have the group scheme $E(G)$ using the conjugation action of $G$ on itself. Let $E(\mathcal{L})$ over $E\left(G_{1} / P_{1}\right)$ be the associated line bundle over the associated rational fiber bundle over $X$.

Let $E(G)_{0}$ be the group scheme defined over the function field $K$ of $X$. We also have the action of $E(G)_{0}$ on the projective variety $E\left(G_{1} / P_{1}\right)_{0} \subset E(\mathbb{P}(V))_{0}$ over $K$, linearized by the line bundle $E(\mathcal{L})_{0}$.

Let $\sigma$ be a reduction of structure group of $E\left(G_{1}\right)$ to $P_{1}$. Let $\sigma_{0}$ be the associated $K$-rational point of $E\left(G_{1} / P_{1}\right)_{0}$. 
In the following two propositions we summarise the basic argument of RamananRamanathan.

Proposition 3.8 Let $\sigma_{0}$ be semistable for the action of $E(G)_{0}$ on $E\left(G_{1} / P_{1}\right)_{0}$ (over $\bar{K})$ for the polarization $E(\mathcal{L})_{0}$. Then the section $\sigma$ has the property that $\operatorname{deg}\left(T_{\sigma}\right) \geq 0$.

Proof See Proposition $(3.10,(1))$ of [20].

Suppose that $\sigma_{0}$ is not semistable for the above action and that the instability parabolic $P^{\prime}$ for $\sigma_{0}$ is defined over the field $K$. Then the parabolic $P^{\prime} \subset E(G)_{0}$ gives rise to a rational reduction of the structure group $\tau$ of $E$ to the parabolic $P$ such that $\left(E_{\tau}(P)\right)_{0}=P^{\prime}$.

The following result is slightly more general than the Proposition (3.13) of [20] (without the semistability assumption on $E$ ) and its proof is along the same lines.

Proposition 3.9 Suppose $\sigma_{0}$ is not semistable and its instability parabolic is defined over the field $K$ then there exists a positive integer $r$ and a dominant character $\chi$ of $P$ (related by the Lemma 3.4) such that the following inequality holds

$$
-\left(r m_{P}\right) \operatorname{deg}\left(T_{\sigma}\right) \leq \operatorname{deg}\left(\chi_{*}\left(E_{\tau}\right)\right)
$$

The above result when $E$ is semistable implies that $\operatorname{deg}\left(T_{\sigma}\right) \geq 0$ and this along with Proposition 3.8 is used in characteristic zero to show that $E\left(G_{1}\right)$ is semistable.

We will use Proposition 3.9 in this generality because the instability parabolic will be defined after a suitable Frobenius pull-back of $E$ which may not be semistable (see proof of Theorem 1.1.).

\section{A result on instability parabolics}

One of the main steps in our proof of Theorem (1.1) is a result (Proposition (4.5)) which gives uniform bounds for the domain of definition of instability parabolics. For proving this result we need to estimate the non-reducedness of the fibers of morphisms of algebraic varieties and we will do this part first.

We start with some definitions which will be used later. Let $K$ be a field and let $\bar{K}$ be its algebraic closure.

We define the radical index $\operatorname{Ri}(A)$ of an affine algebra $A$ over $K$ to be the smallest integer $n$ such that for any $f$ in the $\operatorname{radical} \operatorname{Rad}(A)$ of $A$ we have $f^{n}=0$. For an affine morphism $f: Y \longrightarrow X$ of finite type $\bar{K}$-schemes we define the radical index $\operatorname{Ri}(x)$ of a point $x \in X$ to be $\operatorname{Ri}\left(Y_{x}\right)$ where $Y_{x}$ is the fiber of $f$ at the point $x \in X$.

Proposition 4.1 Let $f: Y \longrightarrow X$ be a morphism of finite type affine schemes over $\bar{K}$. There exists an integer $n$ such that $\operatorname{Ri}(x) \leq n$ for each $x \in X$. 
Proof The proof of this proposition is a series of reductions from the case of arbitrary $X$ and $Y$ to very specific ones using the induction on the dimension of $X$. Let $X=\operatorname{Spec}(A)$ and $Y=\operatorname{Spec}(B)$ and $i$ be the homomorphism $A \longrightarrow B$. For any prime ideal $\mathfrak{p} \in \operatorname{Spec}(A)$ (or $\operatorname{Spec}(B))$ we write $\operatorname{Ri}(\mathfrak{p}, B)$ for the radical index of $B / \mathfrak{p} B$.

First we may assume that $A$ is integral. This part is an elementary check.

We use induction on the $\operatorname{dim}(A)$. So, successively we reduce to the situations where we need to bound $\operatorname{Ri}(\mathfrak{m}, B)$ for maximal ideals $\mathfrak{m} \in \operatorname{Spec}\left(A_{f}\right)$ for suitable choices of $f \in A$.

We make some reductions on $B$. We may assume that $B$ is reduced. For this if $\mathfrak{m}$ is a maximal ideal of $A$ then we can check that

$$
\operatorname{Ri}(\mathfrak{m}, B) \leq \operatorname{Ri}\left(\mathfrak{m}, B_{\text {red }}\right)+\operatorname{Ri}(B)
$$

Next we may assume that $B$ is irreducible. Let $\mathfrak{p}_{i}$, for $i=1 \ldots m$, be the set of minimal prime ideals in $B$. Let $\mathfrak{m}$ be a maximal ideal in $A$. We will show that

$$
\operatorname{Ri}(\mathfrak{m}, B) \leq m \operatorname{Max}_{i=1}^{m} \operatorname{Ri}\left(\mathfrak{m}, B / \mathfrak{p}_{i}\right)
$$

For this one observes that if $x \in \operatorname{Rad}(B / \mathfrak{m} B)$ then the image of $x$ in each of $B / \mathfrak{p}_{i} \otimes$ $A / \mathfrak{m}$ lies in $\operatorname{Rad}\left(\left(B / \mathfrak{p}_{i}\right) / \mathfrak{m}\left(B / \mathfrak{p}_{i}\right)\right)$. Hence if $n=\operatorname{Max}_{i=1}^{m} \operatorname{Ri}(\mathfrak{m}, B / \mathfrak{p})$ then $x$ has the property that $x^{n} \in \cap_{i=1}^{m}\left(\mathfrak{p}_{i}+\mathfrak{m} B\right)$. We can write $x^{n}=y_{i}+z_{i}$ such that $y_{i} \in \mathfrak{p}_{i}$ and $z_{i} \in \mathfrak{m} B$. Since $B$ is reduced we have $\prod_{i=1}^{m}\left(x^{n}-z_{i}\right)=\Pi y_{i}=0$, and so $x^{n m} \in \mathfrak{m} B$. This proves the assertion.

Hence from now on we may assume that $A$ and $B$ are integral domains.

We reduce this problem to an open subscheme of $Y=\operatorname{Spec}(B)$. Let $b \in B$. Then there exists an element $a \in A$ such that $(B / b B)_{a}$ is flat over $A_{a}$. Hence for any maximal ideal $\mathfrak{m}$ in $A_{a}$ we have $\operatorname{Tor}_{1}^{A}\left((B / b B)_{a}, A / \mathfrak{m}\right)=0$.

Consider the map $B_{a} \longrightarrow B_{a b}$. We will show that $\operatorname{Ri}\left(\mathfrak{m}, B_{a}\right) \leq \operatorname{Ri}\left(\mathfrak{m}, B_{a b}\right)$. If $x \in B$ is such that some power of it lies in $B_{a} / \mathfrak{m} B_{a}$ then the image of $x$ also has the same property over $B_{a b}$. Then by clearing denominators if $r=\operatorname{Ri}\left(\mathfrak{m}, B_{a b}\right)$ then there is an $m$ such that $b^{m} x^{r} \in \mathfrak{m} B_{a}$. Using the exact sequence

$$
\cdots \longrightarrow \operatorname{Tor}_{1}^{A}\left((B / b B)_{a}, A / \mathfrak{m}\right) \longrightarrow B_{a} / \mathfrak{m} B_{a} \longrightarrow B_{a} / \mathfrak{m} B_{a}
$$

with the last map being multiplication by $b$, we conclude, by the vanishing of the $\operatorname{Tor}_{1}^{A}\left((B / b B)_{a}, A / \mathfrak{m}\right)$, that $x^{r} \in \mathfrak{m} B_{a}$. Hence it is enough to bound radical index of fibers of some open set of the type $B_{b}$ over $A$.

We use Noether Normalization (and inverting an element of $A$ ) to get an inclusion $A \hookrightarrow A\left[x_{1}, \ldots, x_{r}\right]=A^{\prime} \hookrightarrow B$ such that $B$ is finite over $A^{\prime}$. Let $K_{A}$ (respectively $K_{B}$ and $K_{A^{\prime}}$ ) be the function fields of $A$ (respectively $B$ and $A^{\prime}$ ).

Let $L$ be the separable closure of $K_{A^{\prime}}$ in $K_{B}$. The extension $L \subset K_{B}$ is purely inseparable. Hence there is an integer $n_{1}$ such that for any $x \in K_{B}$, we have $x^{p^{n_{1}}} \in L$. 
Let $C=B \cap L$. Then we observe that $B$ is integral over $C$ and is a finitely generated $A$-algebra. This implies that $C$ is also a finitely generated $A$-algebra. Again by localizing $A$ at an element we can assume that the $A$ module $B / C$ is flat over $A$ and hence for any $\mathfrak{m}$ in $A$ we have $\operatorname{Tor}_{1}^{A}(B / C, A / \mathfrak{m})=0$. This has the effect that for each maximal ideal $\mathfrak{m}$ in $A$ we have an injection $C / \mathfrak{m} C \longrightarrow B / \mathfrak{m} B$. Using this and the fact that $x^{p^{n_{1}}} \in C$ for any $x \in B$ we have

$$
\operatorname{Ri}(\mathfrak{m}, B) \leq \operatorname{Ri}(\mathfrak{m}, C)+p^{n_{1}}
$$

The problem now reduces to proving the proposition for the case when $A \hookrightarrow B$ is an extension of finitely generated domains such that the function field extension is separable. Further, it is enough to prove the result for the case $A \hookrightarrow B_{b}$ for some $b \in B$.

We may now assume that $A$ and $B$ are smooth domains. Hence we conclude that there exist elements $b \in B$ and $a \in A$ such that the morphism $\operatorname{Spec}\left(B_{a b}\right) \longrightarrow$ $\operatorname{Spec}\left(A_{a}\right)$ is smooth. This implies that the fibers here are reduced and hence proof of the proposition is complete.

Let $T$ be a finite type scheme over $\bar{K}$. Let $\mathcal{N}$ be its radical ideal sheaf. This has the property that for any closed point of $T$, the stalk of $\mathcal{N}$ is the radical of the local ring. We define the radical index $\operatorname{Ri}(\mathrm{T})$ of $T$ to be the smallest integer $n$ such that for any open subset $U \subset T$ and for any $g \in \Gamma(U, \mathcal{N})$ we have $g^{n}=0 \in \Gamma\left(U, \mathcal{N}^{n}\right)$. Let $f: Y \longrightarrow X$ be a morphism of finite type $\bar{K}$-schemes. In this general setting we define the radical index of the closed point $x \in X$ by $\operatorname{Ri}(x)=\operatorname{Ri}\left(Y_{x}\right)$, where $Y_{x}$ is the fiber at $x$. In this case we have the following result which generalizes Proposition (4.1) and this will also be used in our proof of main results.

Proposition 4.2 Let $f: Y \longrightarrow X$ be a morphism of finite type schemes over $\bar{K}$ There exists an integer $n$ such that $\operatorname{Ri}(x) \leq n$ for all closed points $x \in X$

Proof Let $\left\{U_{i}\right\}_{i=1}^{r}$ be an open cover of $X$ by affine open subschemes with $U_{i}=$ $\operatorname{Spec}\left(A_{i}\right)$. Then it is enough to prove the result for the case of each of $U_{i}$, hence we may assume that $X=\operatorname{Spec}(A)$ is an affine scheme.

Let $\left\{V_{i}\right\}_{i=1}^{r^{\prime}}$ be an open cover of $Y$ by a finite number of affine open subschemes. We write $V_{i}=\operatorname{Spec}\left(B_{i}\right)$. By Proposition (4.1), we have positive integers $n_{i}$ such that for each maximal ideal $\mathfrak{m}$ of $A$ we have $\operatorname{Ri}\left(\mathfrak{m}, B_{i}\right) \leq n_{i}$. Let $n=\operatorname{Max}\left\{n_{i}\right\}$.

Then we have $\operatorname{Ri}(x, Y) \leq n$ for each closed point $x \in X$. This is because the fiber $Y_{x}$ can be covered by affine open subschemes $\left\{V_{i, x}\right\}$. Here $V_{i, x}$ is the fiber of $x$ in $V_{i}$. If $U \longrightarrow Y_{x}$ is any open immersion and $\nu \in \Gamma(U, \mathcal{N})$ then restriction $\nu_{i}$ of $\nu$ to $U \cap V_{i, x}$ lies in $\Gamma\left(U \cap V_{i, x}, \mathcal{N}\right)$. Hence the result would follow if we show that for any $K$-algebra $B$ and an element $b \in B$ we have $\operatorname{Ri}\left(B_{b}\right) \leq \operatorname{Ri}(B)$. The last statement is a straight forward verification. This completes the proof of Proposition (4.2).

Remark 4.3 One notes that the constant $n$ as defined in the above proposition depends on $X, Y$, and $f$ but not on the $\bar{K}$-valued points of $X$. 
Let $G$ be a reductive algebraic group acting on a variety $M$ (over $\bar{K}$ ). For any $x \in M(\bar{K})$ we denote the isotropy subgroup scheme at $x$ by $G_{x}$. The following result is a consequence of the above proposition.

Proposition 4.4 There exists an $N_{1}$ such that $\operatorname{Ri}\left(G_{x}\right) \leq N_{1}$ for each $x \in M(\bar{K})$.

Proof Consider the map $G \times M \longrightarrow M \times M$ defined by $\left(\rho, p r_{2}\right)$ where $\rho$ is the action map and $p r_{2}$ is the second projection map. Let $\Delta_{M}$ be the diagonal map $M \longrightarrow M \times M$. Let $H=(G \times M) \times_{M \times M} M$. Then we have a natural projection map $\pi: H \longrightarrow M$ which has the property that for any $x \in M(\bar{k})$ the fiber of the map $\pi$ at $x$ is the isotropy subscheme $G_{x}$. The result follows from Proposition (4.2).

Let $K$ be an arbitrary field and $K_{s}$ and $\bar{K}$ be its separable closure and the algebraic closure respectively (in fact $K$ will be the function field of the smooth projective variety $X)$.

In this case the radical index of a finite type scheme $T$ over $K$ is defined to be the radical index of the scheme $\bar{T}=T \otimes_{K} \bar{K}$.

Let $G$ be a reductive group over $K$. Let $M$ and $V$ be as defined before (in Section $3)$.

Let $m$ be a non-semistable $K$ valued point of $M$. Let $P(m)$ be the instability parabolic defined over $\bar{K}$. Recall from Remark (3.5) that if $P(m)$ is defined over $K_{s}$ then it is already defined over $K$. Hence $P(m)$ is always defined over a finite purely inseparable extension of $K$.

The following Proposition is the main result of this Section.

Proposition 4.5 There exists an integer $N$ such that for any $K$-rational point $m$ of $M$ which is not semistable, the instability flag $P(m)$ is defined over $K^{p^{-N}}$.

Proof It is enough to show that there exists an $N$ such that the instability parabolic for any non-semistable $K_{s}$ rational point of $M$ is defined over $K_{s}^{p^{-N}}$ (see Remark (3.5). This enables us to assume that all our objects are defined over the field $K_{s}$.

Let $m$ be a $K_{s}$-valued point of $M$ which is not semistable for the action of $G$ on $M$. Let $O(m)$ be the (reduced) orbit of $G$ at $m$. Let $P(m)$ be its instability parabolic over $\bar{K}$. We can find a $g \in G$ such that $g P(m) g^{-1}=P$ is defined over $K_{s}$ (this was seen before). If $x_{m}=g . m$ then $P=P\left(x_{m}\right)$ is the instability flag of $x_{m}$.

To this setup we have the scheme $M(P)_{x_{m}}$ as defined in Section 3 satisfying the properties of the Lemmas (3.6) and (3.7).

Our main goal is to estimate the non-reducedness of the scheme $M(P)_{x_{m}}$ independent of $m$, and this will enable us to prove that the parabolic $P(m)$ is defined over a fixed purely inseparable extension of $K_{s}$ for all $m$. 
Note that we have made a choice of $x_{m}$ and this choice fixes the instability 1-PS $\lambda$ of $x_{m}$ which is defined over $K_{s}$. Hence the point $x_{m}$ determines the vector subspace $V^{j} \subset V$ (as defined in Section 3) .

We will show that there exists a positive integer $N_{2}$ such that for any non-semistable point $m \in M$ and any choice of $x_{m}$ as above, the radical index $\operatorname{Ri}\left(M(P)_{x_{m}}\right) \leq N_{2}$. The basic idea of the proof is to prove that the spaces $M(P)_{x_{m}}$ occurs as suitable subschemes of the fibers of a fixed morphism $Y \longrightarrow X$ and then apply Proposition (4.2) to bound the radical index. For this analysis we may assume that we are working over the algebraic closure $\bar{K}$ of $K$.

Consider the map $\widetilde{\rho}: G \times \mathbb{P}(V) \longrightarrow \mathbb{P}(V) \times \mathbb{P}(V)$ defined by $\widetilde{\rho}=\left(\rho, \mathrm{pr}_{2}\right)$, where $\rho$ is the action map and $\operatorname{pr}_{2}$ is the second projection. Let $\mathcal{Y}$ be the schematic image of $\widetilde{\rho}$. In this case $\mathcal{Y}$ gets the reduced induced scheme structure from the product and its points are the closure of the image of the map $\widetilde{\rho}$. We have the map $h: \mathcal{Y} \longrightarrow \mathbb{P}(V)$ which is the composition of the inclusion map to $\mathbb{P}(V) \times \mathbb{P}(V)$ with the second projection. Let $\mathcal{Y}_{x}$ be the fiber of $h$ at a point $x \in \mathbb{P}(V)$. One observes that $\mathcal{Y}_{x}$ contains the closure of the orbit $O(x)$ of $x$.

For any integral locally closed subscheme $Z \subset \mathbb{P}(V)$ we have the restriction of the map $\widetilde{\rho}$ which we denote by $\widetilde{\rho}^{Z}$ from $G \times Z \longrightarrow \mathbb{P}(V) \times Z$. Let $\mathcal{Y}^{Z}$ be its schematic image. We again get the induced map $h^{Z}: \mathcal{Y}^{Z} \longrightarrow Z$ and we denote by $\mathcal{Y}_{x}^{Z}$ the fiber of the surjective map $h^{Z}$ at $x \in Z$.

Note that since $Z$ is reduced we have an open subscheme $U_{1} \subset Z$ where the map $h^{Z}$ is flat. One also observes that the schematic image of $\left.\widetilde{\rho}^{Z}\right|_{\left(G \times U_{1}\right)}$ is exactly $\left(h^{Z}\right)^{-1}\left(U_{1}\right)$. Hence we can restrict the setup to $U_{1}$.

Since the actual image set $\mathcal{Z}^{\prime}=\widetilde{\rho}^{Z}\left(G \times U_{1}\right)$ is constructible, it contains an open subset $U^{\prime} \subset \mathcal{Y}^{U_{1}}$. The map $U^{\prime} \longrightarrow U_{1}$ is flat, hence if we define $U=U^{\prime} \cap U_{1}$ and restrict the whole setup to $U$, the image set $\mathcal{Z}=\widetilde{\rho}^{Z}(G \times U)$ contains an open subset $U^{\prime} \subset Y^{U}$ which maps surjectively onto $U$. Now we can further translate the open set $U^{\prime}$ by an element of $g$ which acts on the first factor to obtain that $\mathcal{Z}$ is open in $\mathcal{Y}^{U}$. The upshot of this analysis is that for any subvariety $Z \subset \mathbb{P}(V)$ we can find an open subscheme $U \subset Z$ such that we can define an open reduced subscheme $\mathcal{Z}^{U}$ of $\mathcal{Y}^{U}$ whose points are exactly the image of the map $\tilde{\rho}^{U}$. This also has the property that $O(x)$ is exactly the reduced part $\left(\mathcal{Z}_{x}^{U}\right)_{\text {red }}$ of the fiber $\mathcal{Z}_{x}^{U}$ at $x \in U$.

Let $0<l<\operatorname{dim}(V)$ be an integer. Let $\operatorname{Gr}_{l}(V)$ be the Grassmannian of $l$-dimensional planes in $\mathbb{P}(V)$. We have a universal subscheme $i: H_{l} \subset \operatorname{Gr}_{l}(V) \times \mathbb{P}(V)$. This has the property that for each $y \in \mathrm{Gr}_{l}(V)$ the inverse image $\left(H_{l}\right)_{y}=i^{-1}(\{y\} \times \mathbb{P}(V)) \subset \mathbb{P}(V)$ is $\mathbb{P}\left(W_{y}\right)$, where $W_{y}$ is the $l$ dimensional subspace of $V$ associated to the point $y$.

Now we consider the relative version of the setup to get a suitable intersection of the orbits with projective subspaces of $\mathbb{P}(V)$. Let $Z$ and $U$ be defined as before.

Let $\widetilde{\rho}_{l}^{U}: \operatorname{Gr}_{l}(V) \times G \times U \longrightarrow \operatorname{Gr}_{l}(V) \times \mathbb{P}(V) \times U$ be the map defined by (id, $\left.\widetilde{\rho}^{U}\right)$. Inside $\operatorname{Gr}_{l}(V) \times \mathbb{P}(V) \times U$ we have two subschemes namely $\operatorname{Gr}_{l}(V) \times \mathcal{Z}^{U}$ and $H_{l} \times U$. Let $Y$ be the scheme theoretic intersection of these schemes. We have a map $f$ : $Y \longrightarrow X=\operatorname{Gr}_{l}(V) \times U$ which is obtained by composing the inclusion map with the product of the first and the last projection map. 
The map $f$ has the property that for any $x \in U$ and $y \in \operatorname{Gr}_{l}(V), M_{y, x}^{\prime}=f^{-1}(y, x)$ is the scheme theoretic intersection of $\mathcal{Z}_{x}^{U}$ and $\mathbb{P}\left(W_{y}\right)$. Hence by Proposition (4.2), there exists an integer $n$ such that $\operatorname{Ri}\left(M_{y, x}^{\prime}\right) \leq n$, for each $(y, x) \in X$.

If $M_{y, x}$ is the schematic intersection of $O(x)$ and $M_{y, x}^{\prime}$ then we have an inclusion $\left(M_{y, x}\right)_{\text {red }} \subset M_{y, x} \subset M_{y, x}^{\prime}$. Hence we conclude that for each $(y, x) \in \operatorname{Gr}_{l}(V) \times U$, we have $\operatorname{Ri}\left(\mathbb{P}\left(W_{y}\right) \cap O(x)\right) \leq n$.

Take $Z=\mathbb{P}(V)$ and we obtain an open subscheme $U$ with a bound $n$ for the radical index of $\mathbb{P}\left(W_{y}\right) \cap O(x)$ for every $x \in U$ and $y \in \operatorname{Gr}_{l}(V)$. Then take the complement of $U$ in $\mathbb{P}(V)$ and so on by induction there exists an integer $n_{l}$ such that $\operatorname{Ri}\left(\mathbb{P}\left(W_{y}\right) \cap O(x)\right) \leq n_{l}$ for each $y \in \operatorname{Gr}_{l}(V)$ and $x \in \mathbb{P}(V)$.

We choose $N_{2}=\operatorname{Max}_{l=1}^{r}\left\{n_{l}\right\}$. Then for any $m \in M\left(K_{s}\right)$ which is not semistable and any choice of $x_{m}$ as before the subscheme $M(P)_{x_{m}}$ occurs as one such $M_{y, x}$ for some $y \in \operatorname{Gr}_{l}(V)$ for some $l$ and $x=x_{m}$. This proves that for all choices of $x_{m}$ and $m$ we have $\operatorname{Ri}\left(M(P)_{x_{m}}\right) \leq N_{2}$.

As a next step in the proof of the Proposition (4.5) we will show that for any $m \in M(K)$ the $K_{s}$-scheme $M(P)_{x_{m}}$ admits a $K_{s}^{p^{-N_{2}}}$-rational point. For this we can first find an affine open subscheme $U_{x_{m}}$ of $M(P)_{x_{m}}$ which is defined over $K_{s}$. As the radical index of $U_{x_{m}}$ is $\leq N_{2}$, the existence of rational points on $U_{x_{m}}$ follows from the Lemma below.

Lemma 4.6 Let $A$ be an affine $K_{s}$-algebra with radical index $\leq p^{n}$. Then $A$ admits a $K_{s}^{p^{-n}}$-rational point.

Proof We will denote by $A_{n}$ the $K_{s}^{p^{-n}}$-algebra $A \otimes_{K_{s}} K_{s}^{p^{-n}}$ and by $\bar{A}$ the $\bar{K}_{s^{-}}$ algebra defined by $A \otimes_{K_{s}} \bar{K}_{s}$. Since the radical $\operatorname{Rad}(A) \otimes_{K_{s}} \bar{K}_{s} \subset \operatorname{Rad}(\bar{A})$ we may assume that $A$ is reduced. We show that the natural inclusion

$$
\operatorname{Rad}\left(A_{n}\right) \otimes_{K_{s}} \bar{K}_{s} \subset \operatorname{Rad}(\bar{A})
$$

is an isomorphism. This will prove that the $K_{s}^{p^{-n}}$-algebra $A_{n} / \operatorname{Rad}\left(A_{n}\right)$ is absolutely reduced and hence will admit a $K_{s}^{p^{-n}}$-rational point.

Let $f \in \operatorname{Rad}(\bar{A})$. Then we can write $f=\sum_{i=1}^{l} f_{i}^{\prime} \otimes a_{i}$ with $f_{i} \in A$ and $a_{i} \in \bar{K}_{s}$. If each of $a_{i} \in K_{s}^{p^{-n}}$ then already we have $f \in \operatorname{Rad}\left(A_{n}\right) \otimes_{K_{s}} \bar{K}_{s}$.

Let $n_{1}>n$ be so chosen that each of $a_{i} \in K_{s}^{p^{-n_{1}}}$. Using the identity $\bar{A}=A_{n} \otimes_{K_{s}^{p^{-n}}}$ $\bar{K}_{s}$, we have an expansion of $f=\sum_{i=1}^{j} f_{i} \otimes b_{i}$ where $f_{i} \in A_{n}$ and $b_{i} \in K_{s}^{p^{-n_{1}}}$ such that $\left\{b_{1}, \ldots, b_{j}\right\}$ is linearly independent over $K_{s}^{p^{-n}}$.

We claim that $b_{i}^{p^{n}}$ 's are linearly independent over $K_{s}$. Suppose that $\sum c_{i} b_{i}^{p^{n}}=0$ with $c_{i} \in K_{s}$. Let $d_{i} \in K_{s}^{p^{-n}}$ be such that $d_{i}^{p^{n}}=c_{i}$. The above implies that $\left(\sum d_{i} b_{i}\right)^{p^{n}}=0$. This proves the claim.

The radical index of $A$ is $n$ we have $f^{p^{n}}=0$ and this gives us $0=f^{p^{n}}=\sum_{i=1}^{j} f_{i}^{p^{n}} \otimes$ $b_{i}^{p^{n}}$. Since $b_{i}^{p^{n}}$ 's are linearly independent over $K_{s}$ and $A$ is flat over $K_{s}$, we have $f_{i}^{p^{n}}=0$. Hence $f_{i} \in \operatorname{Rad}\left(A_{n}\right)$. This proves the lemma (4.6).

We have the orbit morphism $G \longrightarrow O(m)$ which is defined over $K_{s}$. Also we have a $K_{s}^{p^{-N_{2}}}$-valued point of $M(P)_{x_{m}}$ which is $K_{s}$-subscheme of $O(m)$. Hence we obtain 
a $K_{s}^{p^{-N_{2}}}$-valued point $y$ of $O(m)$. Let $G_{m}$ be the isotropy subgroup scheme of $G$ at $m$. Then we have a $K_{s}$ isomorphism $G / G_{m} \cong O(m)$. Let $N_{1}$ be as defined in Proposition (4.4). We show that if $N=N_{1}+N_{2}$ then the instability parabolic $P(m)$ of $m$ is defined over $K_{s}^{p^{-N}}$. For this we first show that there is a $K_{s}^{p^{-N}}$-valued point $h$ of $G$ such that it maps to $y$. This statement follows from the fact that if $Y$ is the fiber of the map $G \longrightarrow O(m)$ at the point $y$, then $Y$ defines a principal $G_{m}$-bundle over $S=\operatorname{Spec}\left(K_{s}^{p^{-N_{2}}}\right)$. Now it follows from Proposition (4.4) that the scheme $Y$ is an finite type affine scheme over $S$ whose radical index is $\leq N_{1}$. Hence by lemma (4.6) we conclude that $Y$ admits a $K_{s}^{p^{-N}}$-rational point. This proves that there is a $K_{s}^{p^{-N}}$-valued point $h$ of $G$ such that $h m=y$.

The Lemma (3.7) now implies that the instability parabolic $P(m)$ of $m$ is defined over $K_{s}^{p^{-N}}$ and this completes the proof of the Proposition (4.5).

\section{The proof of the main Theorems}

In this Section we will prove the main Theorems (1.1) and (1.2) stated in the introduction. The basic strategy of the proof is to assume (1.1) for the case of lower semisimple rank groups and prove (1.2). Finally prove the Theorem (1.1) using (1.2) and the Proposition (4.5).

\section{Proof of Theorem (1.2)}

(Assuming Theorem (1.1) for lower semisimple rank)

We fix the Borel subgroup $B$ and a maximal torus $T \subset B$ and the root datum as before. Let $\Delta$ denote the set of simple roots. Let $Q_{\alpha}$ be the maximal parabolic subgroup of $G$ containing $B$ corresponding to the simple root $\alpha \in \Delta$. We will denote by $\mathfrak{q}_{\alpha}$ and $\mathfrak{g}$ the lie algebras of $Q_{\alpha}$ and $G$ respectively. Let $P$ be a parabolic subgroup of $G$ containing $B$. Let $L$ be its Levi quotient. We will use the following lemma which is proved in Biswas-Gomez (see proof of Theorem 4.1, in page 783, [5]) .

Lemma 5.1 Let $P \subset Q_{\alpha}$ be an inclusion of parabolic subgroups. There exists a filtration

$$
0=V_{0}^{\alpha} \subset V_{1}^{\alpha} \subset \ldots \subset V_{a_{\alpha}}^{\alpha}=\mathfrak{g} / \mathfrak{q}_{\alpha}
$$

of $P$ modules such that the following holds.

1. The unipotent radical $R_{u}(P)$ acts trivially on each successive quotients $W_{j}^{\alpha}=$ $V_{j}^{\alpha} / V_{j-1}^{\alpha}$ and (hence) the identity connected component of the center of the Levi quotient $L$ acts by a scalar on the induced representation (denoted by $\rho_{j}^{\alpha}$ ) of $L$.

2. The character $\chi_{j}^{\alpha}$ of $P$ on the action of $P$ on $W_{j}^{\alpha}$ has the property that its restriction to the maximal torus $T$ can be written as a non-positive linear combination of simple roots $\sum_{\beta \in \Delta} n_{j, \beta}^{\alpha} \beta$ with $n_{j, \beta}^{\alpha} \leq 0$ and $n_{j, \alpha}^{\alpha}<0$. 
Let $\Pi$ be the subset of simple roots defined by the property that $\alpha \in \Pi$ if and only if $P \subset Q_{\alpha}$. Here we can look at $P$ as $P_{\Pi}$.

For an $\alpha \in \Pi$, let $\chi_{0}^{\alpha}$ be the character of $P$ which is defined by the representation of $P$ on $\mathfrak{g} / \mathfrak{q}_{\alpha}$. One can check that the restriction of $\chi_{0}^{\alpha}$ to the maximal torus is a non-positive linear combination of simple roots with the coefficients of $\alpha$ being negative. Let $\chi_{P}$ be the character of $P$ defined by the representation of $P$ on $\mathfrak{g} / \mathfrak{p}$.

Let $\mathcal{T}$ be the finite set $\Pi_{\alpha \in \Pi}\left[0, a_{\alpha}\right)$. For an element $\bar{z} \in \mathcal{T}$, the Lemma (5.1) and the observation above imply that there exists positive integers $n(\bar{z})$ and $m^{\alpha}(\bar{z})$ (for each $\alpha \in \Pi$ ) with the property that the restriction of the character $n(\bar{z}) \chi_{P}-$ $\sum_{\alpha \in \Pi} m^{\alpha}(\bar{z}) \chi_{\bar{z}_{\alpha}}^{\alpha}$ to the maximal torus $T$ is a linear combination of simple roots in $\Delta-\Pi$. This automatically implies that

$$
n(\bar{z}) \chi_{P}=\sum_{\alpha \in \Pi} m^{\alpha}(\bar{z}) \chi_{\bar{z}_{\alpha}}^{\alpha}
$$

We will define the constant $N_{P}$ by

$$
N_{P}=\operatorname{Max}_{\bar{z} \in T}\left\{\frac{1}{n(\bar{z})} \sum_{\alpha \in \Pi} m^{\alpha}(\bar{z})\right\}
$$

Lemma (5.1) also implies that representation $\rho_{j}^{\alpha}$ of $L$ takes the identity connected component of the center of $L$ to connected component of the center of $G L\left(W_{j}^{\alpha}\right)$. Hence by the induction assumption (on Theorem 1.1) there exists a constant $C^{L}\left(X, \rho_{j}^{\alpha}\right)$ such that for any rational semistable $L$-bundle $E_{L}$ we have

$$
\mu_{\max }\left(E_{L}\left(W_{j}^{\alpha}\right)\right)-\mu_{\min }\left(E_{L}\left(W_{j}^{\alpha}\right)\right) \leq C^{L}\left(X, \rho_{j}^{\alpha}\right)
$$

Let $C_{P}=\operatorname{Max}\left\{C^{L}\left(X, \rho_{j}^{\alpha}\right)\right\}$, where the maximum is taken over all $\alpha \in \Pi$ and $1 \leq j<a_{\alpha}$.

We also define the constant $M_{P}$ by setting $M_{P}=\operatorname{Max}_{\alpha \in \Pi, j}\left\{\operatorname{dim}\left(W_{j}^{\alpha}\right)\right\}$

Note that the constants $N_{P}, M_{P}$ and $C_{P}$ depend on the parabolic $P$ and since there are only finitely many choices of parabolic subgroups containing $B$, we will define the constant $N$ ( respectively $M$ and $C$ ) to be the maximum of each $N_{P}$ (respectively $M_{P}$ and $C_{P}$ ) over all parabolics containing the Borel subgroup $B$.

We take a rational $G$-bundle $E$ over $X$. Let $\operatorname{Ideg}(E)$ be its instability degree. Let $\left(P^{\prime}, \sigma^{\prime}\right)$ be a Harder-Narasimhan reduction. One notes here that the reduction $\sigma^{\prime}$ satisfies the properties (1) and (2) stated in Section 2.

Let $F=F^{*}(E)$ be the Frobenius pull-back of $E$. Let $\sigma$ be its Harder-Narasimhan reduction to a parabolic $P$ containing $B$. We will denote by $F_{\sigma}$ the $P$-bundle defined by $\sigma$ and $T_{\sigma}$ the tangent bundle along the fibers of $X$. We will denote by $F_{\sigma, L}$ the $L$-bundle obtained by extension of $F_{\sigma}$ to $L$.

We need to bound the slope of $T_{\sigma}$ in terms of the slope of $T_{\sigma^{\prime}}$.

For each $\alpha \in \Pi$, we have an inclusion $P \subset Q_{\alpha}$. This gives rise to a reduction $\sigma_{\alpha}$ of $F$ to $Q_{\alpha}$ and we will denote by $F_{\sigma_{\alpha}}$ the $Q_{\alpha}$-bundle determined by this reduction. 
By Lemma (5.1) we have representations of $P$ in $\mathfrak{g} / \mathfrak{q}_{\alpha}$ and representations $\rho_{j}^{\alpha}$ for $1 \leq j<a_{\alpha}$. These give rise to a vector bundle $T_{\sigma_{\alpha}}$ and a filtration $F_{\sigma}\left(V_{j}^{\alpha}\right)$ of $T_{\sigma_{\alpha}}$ with the property that successive quotients are isomorphic to $F_{\sigma, L}\left(W_{j}^{\alpha}\right)$.

The bundle $F$ (being the Frobenius pull-back) admits a $p$-connection $\nabla$ satisfying the properties defined in Proposition (2.5).

We apply this to the reduction $\sigma_{\alpha}$ to get the map of vector bundles $\nabla_{\sigma_{\alpha}}: T_{X} \longrightarrow T_{\sigma_{\alpha}}$. First we consider the case when the above map is zero. Then there is a reduction $\widetilde{\sigma}$ of $E$ to $Q_{\alpha}$ such that $\sigma_{\alpha}=F^{*}(\widetilde{\sigma})$. This has the effect that $\operatorname{deg}\left(T_{\sigma_{\alpha}}\right)=p \operatorname{deg}\left(T_{\widetilde{\sigma}}\right)$. Hence we have the inequality

$$
\operatorname{deg}\left(T_{\sigma_{\alpha}}\right) \geq p \operatorname{Ideg}(E)
$$

Now suppose that map $\nabla_{\sigma_{\alpha}}$ is not zero. Then there is a $j$ such that the image of $\nabla_{\sigma_{\alpha}}$ is contained in $F_{\sigma}\left(V_{j}^{\alpha}\right)$ and not in $F_{\sigma}\left(V_{j-1}^{\alpha}\right)$. Hence we get a non-trivial map $T_{X} \longrightarrow F_{\sigma, L}\left(W_{j}^{\alpha}\right)$. This implies that $\mu_{\min }\left(T_{X}\right) \leq \mu_{\max }\left(F_{\sigma, L}\left(W_{j}^{\alpha}\right)\right)$.

Let $C_{X}=\mu_{\min }\left(T_{X}\right)$. This combined with equation (15) and the fact that $C_{X}-C$ can be made to be negative implies that

$$
\operatorname{deg}\left(F_{\sigma, L}\left(W_{j}^{\alpha}\right)\right) \geq\left(C_{X}-C\right) M
$$

Further the right hand side in the inequalities (66) and (17), being negative, can be summed up to get a common right hand side, namely $p \operatorname{Ideg}(E)+\left(C_{X}-C\right) M$

Hence for any $\alpha \in \Pi$ either the inequality (6) holds or the inequality (7) holds for some choice of $j$. This implies that if we vary $\alpha \in \Pi$ we obtain an element $\bar{z} \in \mathcal{T}$. Hence for the element $\bar{z}$ using the formula (3) we get

$$
n(\bar{z}) \operatorname{deg}\left(T_{\sigma}\right) \geq \sum_{\alpha \in \Pi}\left(m^{\alpha}(\bar{z})\right)\left(p \operatorname{Ideg}(E)+\left(C_{X}-C\right) M\right)
$$

This implies that $\operatorname{deg}\left(T_{\sigma}\right) \geq N\left(p \operatorname{Ideg}(E)+\left(C_{X}-C\right) M\right)$. This completes the proof of Theorem (1.2).

The above theorem and an induction argument also proves the following.

Corollary 5.2 There exists constants $C$ and $N$ (independent of $E$ ) such that

$$
\operatorname{Ideg}\left(\left(F^{n}\right)^{*} E\right) \geq p^{n} N \operatorname{Ideg}(E)+C
$$

\section{Proof of the Theorem (1.1)}

We fix a Borel subgroup $B_{1}$ of $\mathrm{GL}(V)$. For a parabolic $P_{1}$ of $\mathrm{GL}(V)$ containing $B_{1}$, we have an action of $G$ on $M_{P_{1}}=\mathrm{GL}(V) / P_{1}$. We fix a representation $\mathrm{GL}(V) \longrightarrow$ $\mathrm{GL}\left(V_{P_{1}}\right)$ such that it defines an embedding of $\mathrm{GL}(V) / P_{1} \subset \mathbb{P}\left(V_{P_{1}}\right)$ with the property that the character of $P_{1}$ on $V_{P_{1}}$ is a positive multiple $m_{P_{1}}$ of the character of $\chi_{P_{1}}$ 
associated to the restriction of the adjoint representation of $P_{1}$ on the vector space $\mathfrak{g} l(V) / \mathfrak{p}_{1}$.

The line bundle $\mathcal{O}(-1)$ on $\mathbb{P}\left(V_{P_{1}}\right)$ when restricted to $\mathrm{GL}(V) / P_{1}$ defines an anti ample line bundle $\mathcal{L}_{P_{1}}^{-1}$ which is also defined by the character $-m_{P_{1}} \chi_{P_{1}}$.

For a rational $G$-bundle $E$ over $X$ we have a rational fiber bundle $E\left(M_{P_{1}}\right)$ and the line bundle $\mathcal{O}(-1)$ gives a rational line bundle $E\left(\mathcal{L}_{P_{1}}^{-1}\right)$.

Let $x_{0}$ be the generic point of $X$. Let $E(G)_{0}$ the group scheme over $K=k(X)$ associated to $E$ at the generic point $x_{0}$. Then we have an action of $E(G)_{0}$ on $E\left(M_{P_{1}}\right)_{0}$ which is linearized with respect to the line bundle $E\left(\mathcal{L}_{P_{1}}\right)_{0}$ over $K$.

Lemma 5.3 There exists a constant $N$, depending only on $G$ and $X$, such that for any rational $G$-bundle $E$ and for any parabolic $P_{1}$ containing $B_{1}$ the instability parabolic for any $K$-valued non-semistable point of $E\left(M_{P_{1}}\right)$ (for the above action of $\left.E(G)_{0}\right)$ is defined over $K^{p^{-N}}$.

Proof Let $E_{0}$ be the principal $G$-bundle over $K$ obtained by restriction of $E$ to the generic point of $X$. One observes that $E_{0}$ becomes trivial over a finite separable extension of $K$, hence when we change the base to $K_{s}$, the separable closure of $K$, we get an isomorphism $E_{0} \otimes_{K} K_{s} \cong G \otimes_{k} K_{s}$. This isomorphism now canonically extends to give an isomorphism of $E(G)_{0} \otimes_{K} K_{s} \cong G \otimes_{k} K_{s}$ and $E\left(M_{P_{1}}\right)_{0} \otimes_{K} K_{s} \cong M_{P_{1}} \otimes_{k} K_{s}$, and the last one being compatible with group actions, and also of the isomorphisms between the ample line bundles on these spaces.

For the induced action of $G \otimes_{k} K_{s}$ on $M_{P_{1}} \otimes_{k} K_{s}$ which is linearised by $\mathcal{L}_{P_{1}} \otimes_{k} K_{s}$, by Proposition (4.5), it follows that there is a positive integer $N$ such that for any nonsemistable point $m$ of $M_{P_{1}} \otimes_{k} K_{s}$ the instability is defined over $K_{s}^{p^{-N}}$. Since there are only finitely many parabolic subgroups containing $B_{1}$ we can find a constant $N$ which works for all these parabolic subgroups.

This implies that the group scheme $E(G)_{0} \otimes_{K} K_{s}$ for any $E$ also has the same property. The Galois descent argument implies that instability parabolic of a $K$ valued point of $E\left(M_{P_{1}}\right)_{0}$ is defined over the field extension $K^{p^{-N}}$, with $N$ being independent of the rational $G$-bundle $E$ and the reduction $\sigma$. This proves the lemma.

A rational reduction $\sigma$ of $\rho_{*} E$ to $P_{1}$ gives a $K$-rational point $\sigma\left(x_{0}\right)$ of $E\left(M_{P_{1}}\right)_{0}$. If this point is semistable then by Proposition (3.8), we have

$$
\operatorname{deg}\left(\sigma^{*} E\left(\mathcal{L}_{P_{1}}\right)\right)=m_{P_{1}} \operatorname{deg}\left(T_{\sigma}\right) \geq 0
$$

If the point $\sigma\left(x_{0}\right)$ is not semistable we have an integer $N$ prescribed by Lemma (5.3) such that its instability parabolic $P_{0}^{\prime}$ is defined over $K^{p^{-N}}$.

One observes that pull-back by the $N$-th Frobenius morphism $F^{N}$ of $X$, the action of the generic fibre $\left(\left(F^{N}\right)^{*} E(G)\right)_{0}=\left(F^{N}\right)_{K}^{*}\left(E(G)_{0}\right)$ on $\left(\left(F^{N}\right)^{*} E\left(M_{P_{1}}\right)\right)_{0}=$ $\left(F^{N}\right)_{K}^{*}\left(E(M)_{0}\right)$ is the base change by the Frobenius $F_{K}^{N}: \operatorname{Spec}(K) \rightarrow \operatorname{Spec}(K)$ of the action of $E(G)_{0}$ on $E(M)_{0}$. Hence the point $\left(\left(F^{N}\right)^{*} \sigma\right)\left(x_{0}\right)$ has an instability parabolic $P_{0}^{\prime}$ for the action of $\left(F^{N}\right)_{K}^{*}\left(E(G)_{0}\right)$ defined over $K^{p^{-N}}$ (see proof of Theorem (3.23) of [20]). 
The parabolic subgroup $P_{0}^{\prime}$ defines a rational reduction of the structure group $\tau$ of $\left(F^{N}\right)^{*} E$ to a parabolic subgroup $P^{\prime} \subset G$ with $P_{0}^{\prime}=\left(E_{\tau}\left(P^{\prime}\right)\right)_{0}$.

Since $P_{0}^{\prime}$ is defined over $K^{p^{-N}}$, by Remark (3.5), the instability 1-PS for $\sigma\left(x_{0}\right)$ is also defined over $K^{p^{-N}}$.

The Proposition (3.9) (applied to bundle $\left(F^{N}\right)^{*} E$ and the point $\left.\left(\left(F^{N}\right)^{*} \sigma\right)\left(x_{0}\right)\right)$ implies that there is a positive integer $r$ and a dominant character $\chi$ of $P^{\prime}$ such that the following inequality holds.

$$
-r \operatorname{deg}\left(\left(\left(F^{N}\right)^{*} \sigma\right)^{*}\left(\left(\left(F^{N}\right)^{*} E\right)\left(\mathcal{L}_{P_{1}}\right)\right)\right) \leq \operatorname{deg}\left(\chi_{*}\left(\left(\left(F^{N}\right)^{*} E\right)_{\tau}\right)\right)
$$

Let $\Pi \subset \Delta$ be the subset defining the parabolic $P^{\prime}$. Let $Q_{\alpha}$ be the maximal parabolic subgroup of $G$ containing $P^{\prime}$ defined by $\alpha$. Let $\chi_{\alpha}$ be the dominant character of $Q_{\alpha}$ defined by the representation $\mathfrak{g} / \mathfrak{q}_{\alpha}$. There is a positive integer $m_{\alpha}$ such that we have $\left.\chi_{\alpha}\right|_{T}=-m_{\alpha} w_{\alpha}$ where $w_{\alpha}$ are the fundamental weights of $G$ with respect to a fixed maximal torus contained in $P^{\prime}$.

Let $L^{\prime}=P^{\prime} / R_{u}\left(P^{\prime}\right)$ and $Z_{0}\left(L^{\prime}\right)$ be the connected component of the center of the Levi $L^{\prime}$.

By Lemma (3.4) we have a character $\chi^{\prime}$ of the maximal torus $T \subset P^{\prime}$ such that $\left.\chi^{\prime}\right|_{Z^{0}\left(L^{\prime}\right)}=\left.\chi\right|_{Z^{0}\left(L^{\prime}\right)}$ and $\chi^{\prime}=r \nu(v, \lambda)\|\lambda\| l_{\lambda}$. Here $l_{\lambda}$ is the dual of $\lambda$.

The above equality can be rewritten as $\chi^{\prime}=r \nu\left(\sigma_{N}, \lambda\right) l_{a}$ where $a \in \mathcal{X}_{*}(T) \otimes \mathbb{Q}$ is the element in the unit sphere defined by $a=\lambda /\|\lambda\|$.

By Lemma (3.1) there exists a constant $B_{G}$ such that for every point $m \in M_{P_{1}} \otimes_{k} \bar{K}$ and $\lambda \in \mathcal{X}_{*}(\mathcal{T}) \otimes \mathbb{Q}$ we have

$$
\nu(m, \lambda) \leq B_{G}
$$

The Weyl group invariant scalar product on $\mathcal{X}_{*}(T) \otimes \mathbb{Q}$ induces a scalar product on $\mathcal{X}^{*}(T) \otimes \mathbb{Q}$. The following lemma is an elementary calculation.

Lemma 5.4 Let $S=\left\{l \in \mathcal{X}^{*}(T) \otimes \mathbb{Q} \mid\|l\|=1\right\}$. Then there exists a constant $A_{G}$ such that for each $l \in S$ if $l=\sum_{\alpha \in \Delta} r_{\alpha} w_{\alpha}$ then $\left|r_{\alpha}\right| \leq A_{G}$ for each $\alpha \in \Delta$.

One notes that under the scalar product we have $\left\|l_{a}\right\|=1$. Since $l_{a}$ is trivial on the center of $G$, the Lemma (5.4) implies that $l_{a}=\sum_{\alpha \in \Delta} r_{\alpha} w_{\alpha}$ with $\left|r_{\alpha}\right| \leq A_{G}$.

This along with the above description of $r$ and $\chi$ we get

$$
\left.\chi\right|_{Z_{0}\left(M^{\prime}\right)}=r \nu\left(\sigma_{N}, \lambda\right) \sum_{\alpha \in \Pi} r_{\alpha} w_{\alpha}
$$

the last equality can be rewritten in terms of $\chi_{\alpha}$ as follows.

$$
-\chi=r \nu\left(\sigma_{N}, \lambda\right) \sum_{\alpha \in \Pi}\left(r_{\alpha} / m_{\alpha}\right) \chi_{\alpha}
$$

Using the fact that $\operatorname{deg}\left(\chi_{\alpha *}\left(\left(\left(F^{N}\right)^{*} E\right)_{\tau}\right)\right) \geq \operatorname{Ideg}\left(\left(F^{N}\right)^{*} E\right)$ and combining it with the inequality (10) we obtain

$$
\operatorname{deg}\left(\chi_{*}\left(\left(\left(F^{N}\right)^{*} E\right)_{\tau}\right)\right) \leq-r B_{G} A_{G}|\Delta| \operatorname{Ideg}\left(\left(F^{N}\right)^{*} E\right) .
$$


This along with (9) implies that there exists a constant $C(G)$ depending only on $G$ such that $\operatorname{deg}\left(\left(\left(F^{N}\right)^{*}(\sigma)\right)^{*} E\left(\mathcal{L}_{P_{1}}\right)\right) \geq C(G) \operatorname{Ideg}\left(\left(F^{N}\right)^{*} E\right)$.

Since $\left(\left(F^{N}\right)^{*} \sigma\right)^{*}\left(\left(\left(F^{N}\right)^{*} E\right)\left(\mathcal{L}_{P_{1}}\right)\right)=\left(\sigma^{*}\left(E\left(\mathcal{L}_{P_{1}}\right)\right)\right)^{p^{N}}$ it follows that $\operatorname{deg}\left(\sigma^{*}\left(E\left(\mathcal{L}_{P_{1}}\right)\right) \geq\right.$ $\left(C(G) / p^{N}\right) \operatorname{Ideg}\left(\left(F^{N}\right)^{*} E\right)$. By (8) we have $\operatorname{deg} T_{\sigma} \geq\left(C(G) /\left(m_{P_{1}} p^{N}\right)\right) \operatorname{Ideg}\left(\left(F^{N}\right)^{*} E\right)$ for every rational reduction $\sigma$ of $\rho_{*} E$ to $P_{1}$ which has the property that $\sigma\left(x_{0}\right)$ is not semistable.

This implies that we have a constant $C(G, \rho)$ such that

$$
\operatorname{Ideg}\left(\rho_{*} E\right) \geq C(G, \rho) \frac{\operatorname{Ideg}\left(\left(F^{N}\right)^{*} E\right)}{p^{N}}
$$

By Corollary (5.2) and Lemma (2.4) we are through with the proof of the Theorem (1.1).

Remark 5.5 The proof actually shows the following more general result. Let $\rho: G \rightarrow G^{\prime}$ be a homomorphism of connected reductive groups which takes the identity connected component of the center of $G$ to the center of $G^{\prime}$. Then there exists constants $C$ and $C^{\prime}$ (depending only on $X, G$, and $\rho$ ) such that for any rational $G$-bundle $E$ over $X$ we have

$$
\operatorname{Ideg}\left(\rho_{*} E\right) \geq C \operatorname{Ideg}(E)+C^{\prime} .
$$

With this formulation, Theorem (1.2) is a special case of this result when applied to the Frobenius homomorphism of $G$.

\section{Boundedness of semistable bundles}

In this section we prove the boundedness of semistable $G$-bundles on $X$ under the assumption stated in the introduction. From now on we work with $G$-bundles on $X$ and not the rational ones.

Let $\mathcal{X}(G)$ be the group of characters of $G$. Let $A^{k}(X)$ be the $k$-th Chow group. To a principal $G$-bundle $E$, recall the definition of the degree $d_{E} \in \operatorname{Hom}\left(\mathcal{X}(G), A^{1}(X)\right)$ of a principal $G$ bundle $E$ from the introduction.

We fix a collection of elements $c_{i} \in A^{i}(X)$ for $2 \leq i \leq n=\operatorname{dim}(X)$ and also we fix an element $d \in \operatorname{Hom}\left(\mathcal{X}(G), A^{1}(X)\right)$.

Under the assumptions in Theorem (1.3), we will show that the set $\mathcal{S}_{G}\left(d ; c_{2}, \ldots, c_{n}\right)$ of isomorphism classes of semistable $G$-bundles $\{E\}$ with degree $d_{E}=d$ and the Chern classes $c_{i}(\operatorname{ad}(E))=c_{i}$ is bounded. Our proof is based on Proposition (4.12) of [19]

We begin with an elementary lemma which allows us to use representations.

Lemma 6.1 There is a faithful completely reducible rational representation of $G$. 
Proof For any irreducible representation $\rho$ of $G$ in a vector space $V$ let $\operatorname{ker}(\rho)$ be the kernel. We first show that $N=\bigcap_{\rho} \operatorname{ker}(\rho)$, over all irreducible representations, is trivial. This is because if $\rho_{1}$ is a faithful representation of $G$ (hence of $N$ ) on a vector space $W$ then there is a filtration of $W$ such that successive quotients are irreducible. This implies that $\rho_{1}(N) \subset \mathrm{GL}(W)$ lies in a parabolic subgroup and its image when composed with the projection to the Levi quotient is trivial. Hence $N$ is a unipotent normal subgroup scheme. Let $N_{0}$ be the identity component of $N$. Since $G$ is connected, using the conjugation map $G \times N_{0} \longrightarrow N$ defined by $(g, n) \mapsto g n g^{-1}$, we check that $N_{0}$ is normal. Again using the conjugation map, this time from $G \times\left(N_{0}\right)_{\text {red }} \longrightarrow N$ we see that $\left(N_{0}\right)_{\text {red }}$ is also normal. Since $G$ is reductive this proves that $N$ is a finite subgroup scheme of $G$. Now using the conjugation map for the third time we get that $N$ is central and hence it is diagonalizable. Now we see that the representation $\rho_{1}$ restricted to $N$ is trivial which is a contradiction.

Now by dimension and length count we can find finitely many irreducible representations $\rho_{i}$, for $i=1, \ldots, m$, of $G$ such that $\bigcap_{i=1}^{m} \operatorname{ker}\left(\rho_{i}\right)=0$. This proves the lemma.

We also have another general lemma.

Lemma 6.2 Let $\rho: \mathrm{GL}(V) \longrightarrow \mathrm{GL}(W)$ be a representation of $\mathrm{GL}(V)$. Let $E_{1}$ and $E_{2}$ be two $\mathrm{GL}(V)$ bundles over $X$ such that $c_{i}\left(E_{1}\right)=c_{i}\left(E_{2}\right)$ for each $i$. Then we have $c_{i}\left(E_{1}(W)\right)=c_{i}\left(E_{2}(W)\right)$ for each $i$.

Proof Let $A^{*}(\operatorname{BGL}(V))$ (respectively $A^{*}(\operatorname{BGL}(W))$ ) be the Chow ring of $\operatorname{BGL}(V)$ (respectively $\mathrm{BGL}(W)$ ). Then one knows that $A^{*}(\operatorname{BGL}(V)) \cong \mathbb{Z}\left[c_{1}, \ldots, c_{n}\right]$ (see [24]). The representation $\rho$ gives rise to the map $\rho^{*}: A^{*}(\operatorname{BGL}(W)) \longrightarrow A^{*}(\operatorname{BGL}(V))$. Now we have the classifying maps $f_{E_{i}}: X \longrightarrow \operatorname{BGL}(V)$ for $i=1,2$. The conditions of the lemma imply that the induced maps $f_{E_{i}}^{*}: A^{*}(\operatorname{BGL}(V)) \longrightarrow A^{*}(X)$ are equal. This implies that the maps $f_{E_{1}}^{*} \circ \rho^{*}=f_{E_{2}}^{*} \circ \rho^{*}$. Hence the Lemma follows.

We continue with the proof of the Theorem (1.3). By lemma (6.1), we have a completely reducible representation $\rho=\bigoplus \rho_{i}$ on $V=\bigoplus V_{i}$ of $G$ which is faithful. Here $V_{i}$ are the irreducible components.

For a fixed $i$, the representation $\rho_{i}$ takes the identity connected component of the center of $G$ to the center of $\mathrm{GL}\left(V_{i}\right)$. Hence for $E$ and $E^{\prime}$ in $\mathcal{S}_{G}\left(d ; c_{2}, \ldots, c_{a}\right)$, we have $c_{1}\left(E\left(V_{i}\right)\right)=c_{1}\left(E^{\prime}\left(V_{i}\right)\right)$.

The representation $\rho_{i}$ induces a Lie algebra homomorphism $\mathfrak{g} \longrightarrow \operatorname{End}\left(V_{i}\right)$. The lemma (6.2) now proves that the two vector bundles $E\left(\operatorname{End}\left(V_{i}\right)\right)=\operatorname{End}\left(E\left(V_{i}\right)\right)$ and $E^{\prime}\left(\operatorname{End}\left(V_{i}\right)\right)=\operatorname{End}\left(E^{\prime}\left(V_{i}\right)\right)$ have same Chern classes. Since the Chern classes of a vector bundle are completely determined by the first Chern class and the Chern classes of the endomorphism bundle we conclude that the Chern classes of $E\left(V_{i}\right)$ are are independent of the individual members $E$ in $\mathcal{S}_{G}\left(d ; c_{2}, \ldots, c_{n}\right)$.

By Theorem (1.1) there are constants $C_{i}=C\left(X, \rho_{i}\right)$ for the representation $\rho_{i}$.

Now the assumptions in Theorem (1.3) imply that there is a finite type scheme $S_{i}$ and a family $U_{i}$ of vector bundles over $S_{i} \times X$ which contains every member of $\mathcal{S}_{C_{i}}\left(r_{i} ; c_{1}\left(E\left(V_{i}\right)\right), \ldots c_{n}\left(E\left(V_{i}\right)\right)\right)$ occurs. 
Now we take the scheme $S=\prod_{i=1}^{m} S_{i}$ and $U=U_{1} \times_{X} U_{2} \times_{X} \ldots U_{m}$. Then Theorem (1.3) follows from the arguments in the last part of the Proposition 3.1 in [10].

Remark 6.3 It should be possible to prove a version of the above Theorem for the case of principal $G$-sheaves in the sense of 8 .

\section{References}

[1] M. F. Atiyah and R. Bott, The Yang-Mills equations over Riemann surfaces, Phil. Trans. Roy. Soc. Lond. A 308 (1982), 523-615.

[2] B. Anchouche, H. Azad and I. Biswas, Harder-Narasimhan reduction for principal bundles over a compact Kähler manifold, Math. Ann. 323 (2002), 693-712.

[3] K. A. Behrend, Semistability of reductive group schemes over curves, Math. Ann. 301 (1995), 281-305.

[4] I. Biswas and Y. Holla, Harder-Narasimhan reduction of a principal bundle, Nagoya Math. J. 174 (2004).

[5] I. Biswas and T. Gomez, Restriction theorems for principal bundles, Math. Ann. 327 (2003), 773-792.

[6] A. Borel and J. Tits, Groupes Réductifs, Pub. Math. I.H.E.S. 27 (1965), $55-150$.

[7] D. Gieseker, On the moduli of vector bundles on an algebraic surface Ann. of Math. (2) 106 (1977), no.1, 45-60

[8] T. Gomez and I. Sols, Moduli space of principal sheaves over projective varieties, Preprint.

[9] G. Harder and M. S. Narasimhan, On the cohomology groups of moduli spaces of vector bundles on curves, Math. Ann. 212 (1975), 215-248.

[10] Y. I. Holla and M. S. Narasimhan, A generalisation of Nagata's theorem on ruled surfaces, Compositio Math. 127 (2001),no. 3, 321-332.

[11] D. Huybrechts and M. Lehn, The geometry of moduli spaces of sheaves, Aspects of Mathematics 311997.

[12] J. Jantzen, Representation of algebraic groups, Academic Press, inc., (1987)

[13] G. Kempf, Instability in invariant theory, Ann. of Math. 108 (1978), 299-316.

[14] A. Langer, Semistable sheaves in positive characteristic, Preprint. 
[15] S. Ilangovan, V. B. Mehta and A. J. Parameswaran, Semistability and semisimplicity in representations of low height in positive characteristic, A tribute to C. S. Seshadri, Perspectives in Geometry and Representation Theory, Hindustan Book Agency (2003), 271-282.

[16] M. Maruyama, Stable vector bundles on an algebraic surface, Nagoya Math.J. 58 (1975), 25-68.

[17] V. B. Mehta and S. Subramanian, On the Harder-Narasimhan filtration of principal bundles, Algebra, Arithmetic and Geometry (Ed. R. Parimala), pp. 405-415. Narosa Pub. House, 2002.

[18] D. Mumford, J. Fogarty and F. Kirwan, Geometric invariant theory, Third edition. Ergebnisse der Mathematik und ihrer Grenzgebiete (2) [Results in Mathematics and Related Areas (2)], 34. Springer-Verlag, Berlin, (1994).

[19] A. Ramanathan, Moduli of principal bundles over algebraic curves II, Proc. Indian Acad. Sci. Math. Sci. 106 (1996), no. 4, 421-449.

[20] S. Ramanan and A. Ramanathan, Some remarks on the instability flag, Tohoku Math. Jour. 36 (1984), 269-291.

[21] A. Ramanathan, Moduli of principal bundles, Algebraic geometry (Proc. Summer Meeting, Univ. Copenhagen, 1978), pp. 527-533, Lecture Notes in Math. 732, Springer, Berlin, 1979.

[22] N. I. Shephard-Barron, Semistability and Reduction mod p, Topology 37 (1998), 659-654.

[23] X. Sun, Remarks on semistability of G-bundles in positive characteristic, Compositio Math. 119 (1999),no. 1, 41-52.

[24] B. Totaro, The Chow ring of a classifying space, Proc. Sympos. Pure Math. Amer. Math. Soc., Providence, RI, 1999, 249-281.

Address :

School of Mathematics, Tata Institute of Fundamental Research, Homi Bhabha Road, Mumbai 400 005, India. e-mail: yogi@math.tifr.res.in,

S.I.S.S.A. Via Beirut 4, 34013 Trieste, Italy

e-mail: coiai@sissa.it 